\title{
On the enumeration of positive cells in generalized cluster complexes and Catalan hyperplane arrangements
}

\author{
Christos A. Athanasiadis · Eleni Tzanaki
}

Received: April 5, 2005 / Revised: October 3, 2005 / Accepted: October 12, 2005

(C) Springer Science + Business Media, LLC 2006

\begin{abstract}
Let $\Phi$ be an irreducible crystallographic root system with Weyl group $W$ and coroot lattice $\check{Q}$, spanning a Euclidean space $V$. Let $m$ be a positive integer and $\mathcal{A}_{\Phi}^{m}$ be the arrangement of hyperplanes in $V$ of the form $(\alpha, x)=k$ for $\alpha \in \Phi$ and $k=0,1, \ldots, m$. It is known that the number $N^{+}(\Phi, m)$ of bounded dominant regions of $\mathcal{A}_{\Phi}^{m}$ is equal to the number of facets of the positive part $\Delta_{+}^{m}(\Phi)$ of the generalized cluster complex associated to the pair $(\Phi, m)$ by S. Fomin and N. Reading.

We define a statistic on the set of bounded dominant regions of $\mathcal{A}_{\Phi}^{m}$ and conjecture that the corresponding refinement of $N^{+}(\Phi, m)$ coincides with the $h$-vector of $\Delta_{+}^{m}(\Phi)$. We compute these refined numbers for the classical root systems as well as for all root systems when $m=1$ and verify the conjecture when $\Phi$ has type $A, B$ or $C$ and when $m=1$. We give several combinatorial interpretations to these numbers in terms of chains of order ideals in the root poset of $\Phi$, orbits of the action of $W$ on the quotient $\check{Q} /(m h-1) \check{Q}$ and coroot lattice points inside a certain simplex, analogous to the ones given by the first author in the case of the set of all dominant regions of $\mathcal{A}_{\Phi}^{m}$. We also provide a dual interpretation in terms of order filters in the root poset of $\Phi$ in the special case $m=1$.
\end{abstract}

Keywords Catalan arrangement · Bounded region · Generalized cluster complex · Positive part $\cdot h$-vector

2000 Mathematics Subject Classification Primary-20F55; Secondary—05E99, 20H15

\footnotetext{
C. A. Athanasiadis $(\bowtie)$

Department of Mathematics (Division of Algebra-Geometry), University of Athens, Panepistimioupolis, Athens 15784, Greece

e-mail: caath@math.uoa.gr

E. Tzanaki

Department of Mathematics, University of Crete 71409 Heraklion, Crete, Greece

e-mail: etzanaki@math.uoc.gr
} 


\section{Introduction and results}

Let $V$ be an $\ell$-dimensional Euclidean space, with inner product (, ). Let $\Phi$ be a (finite) irreducible crystallographic root system spanning $V$ and $m$ be a fixed nonnegative integer. We denote by $\mathcal{A}_{\Phi}^{m}$ the collection of hyperplanes in $V$ defined by the affine equations $(\alpha, x)=k$ for $\alpha \in \Phi$ and $k=0,1, \ldots, m$, known as the $m$ th extended Catalan arrangement associated to $\Phi$. Thus $\mathcal{A}_{\Phi}^{m}$ is invariant under the action of the Weyl group $W$ associated to $\Phi$ and reduces to the Coxeter arrangement $\mathcal{A}_{\Phi}$ for $m=0$. Let $\Delta^{m}(\Phi)$ denote the generalized cluster complex associated to the pair $(\Phi, m)$ by S. Fomin and N. Reading [7]. This is a simplicial complex which reduces to the cluster complex $\Delta(\Phi)$ of S. Fomin and A. Zelevinsky [9] when $m=1$. It contains a natural subcomplex, called the positive part of $\Delta^{m}(\Phi)$ and denoted by $\Delta_{+}^{m}(\Phi)$, as an induced subcomplex. The complex $\Delta^{m}(\Phi)$ was also studied independently by the second author [18] when $\Phi$ is of type $A$ or $B$; see Section 2 for further information and references.

The Weyl group $W$ acts on the coroot lattice $\check{Q}$ of $\Phi$ and its dilate $(m h-1) \check{Q}$, where $h$ denotes the Coxeter number of $\Phi$. Hence $W$ acts also on the quotient $T_{m}=\check{Q} /(m h-1) \check{Q}$. For a fixed choice of a positive system $\Phi^{+} \subseteq \Phi$, consider the partial order on $\Phi^{+}$defined by letting $\alpha \leq \beta$ if $\beta-\alpha$ is a nonnegative linear combination of positive roots, known as the root poset of $\Phi$. An order filter or dual order ideal in $\Phi^{+}$is a subset $\mathcal{I}$ of $\Phi^{+}$such that $\alpha \in \mathcal{I}$ and $\alpha \leq \beta$ in $\Phi^{+}$imply $\beta \in \mathcal{I}$. The filter $\mathcal{I}$ is called positive if it does not contain any simple root.

The following theorem connects the objects just discussed. Parts (i), (ii) and (iii) appear in [1, Corollary 1.3], [7, Proposition 2.13] and [10, Theorem 7.4.2], respectively. The last statement was found independently in $[1,13,16]$.

Theorem 1.1. ([1, 7, 10]) Let $\Phi$ be an irreducible crystallographic root system of rank $\ell$ with Weyl group $W$, Coxeter number $h$ and exponents $e_{1}, e_{2}, \ldots, e_{\ell}$. Let $m$ be a positive integer and let

$$
N^{+}(\Phi, m)=\prod_{i=1}^{\ell} \frac{e_{i}+m h-1}{e_{i}+1} .
$$

The following are equal to $N^{+}(\Phi, m)$ :

(i) the number of bounded regions of $\mathcal{A}_{\Phi}^{m}$ which lie in the fundamental chamber of $\mathcal{A}_{\Phi}$,

(ii) the number of facets of $\Delta_{+}^{m}(\Phi)$ and

(iii) the number of orbits of the action of $W$ on $\check{Q} /(m h-1) \check{Q}$.

Moreover, for $m=1$ this number is equal to the number of positive filters in the root poset of $\Phi$.

The purpose of this paper is to define and study a refinement of the number $N^{+}(\Phi, m)$ and prove that it has similar properties with the one defined by the first author [2] for the total number

$$
N(\Phi, m)=\prod_{i=1}^{\ell} \frac{e_{i}+m h+1}{e_{i}+1}
$$

of regions of $\mathcal{A}_{\Phi}^{m}$ in the fundamental chamber of $\mathcal{A}_{\Phi}$. To be more precise let $H_{\alpha, k}$ be the affine hyperplane in $V$ defined by the equation $(\alpha, x)=k$ and $A_{\circ}$ be the fundamental alcove of the affine Weyl arrangement corresponding to $\Phi$. A wall of a region $R$ of $\mathcal{A}_{\Phi}^{m}$ is a hyperplane in $V$ which supports a facet of $R$. For $0 \leq i \leq \ell$ we denote by $h_{i}(\Phi, m)$ the number of regions $R$ of $\mathcal{A}_{\Phi}^{m}$ in the fundamental chamber of $\mathcal{A}_{\Phi}$ for which exactly $\ell-i$ walls of $R$ of the form 且 Springer 
$H_{\alpha, m}$ separate $R$ from $A_{\circ}$, meaning that $(\alpha, x)>m$ holds for $x \in R$. The numbers $h_{i}(\Phi, m)$ were introduced and studied in [2]. Let $h_{i}\left(\Delta^{m}(\Phi)\right)$ and $h_{i}\left(\Delta_{+}^{m}(\Phi)\right)$ be the $i$ th entries of the $h$-vector of the simplicial complexes $\Delta^{m}(\Phi)$ and $\Delta_{+}^{m}(\Phi)$, respectively. It follows from case by case computations in $[2,7,18]$ that $h_{i}(\Phi, m)=h_{i}\left(\Delta^{m}(\Phi)\right)$ for all $i$ when $\Phi$ is of classical type in the Cartan-Killing classification.

We define $h_{i}^{+}(\Phi, m)$ as the number of bounded regions $R$ of $\mathcal{A}_{\Phi}^{m}$ in the fundamental chamber of $\mathcal{A}_{\Phi}$ for which exactly $\ell-i$ walls of $R$ of the form $H_{\alpha, m}$ do not separate $R$ from the fundamental alcove $A_{\circ}$. Theorem 1.1 implies that the sum of the numbers $h_{i}^{+}(\Phi, m)$, as well as that of $h_{i}\left(\Delta_{+}^{m}(\Phi)\right)$, for $0 \leq i \leq \ell$ is equal to $N^{+}(\Phi, m)$. The significance of the numbers $h_{i}^{+}(\Phi, m)$ comes from the following conjecture, which can be viewed as the positive analogue of [7, Conjecture 3.1].

Conjecture 1.2. For any irreducible crystallographic root system $\Phi$ and all $m \geq 1$ and $0 \leq$ $i \leq \ell$ we have $h_{i}^{+}(\Phi, m)=h_{i}\left(\Delta_{+}^{m}(\Phi)\right)$.

Our first main result (Corollary 5.5) establishes the previous conjecture when $m=1$ and when $\Phi$ has type $A, B$ or $C$ and $m$ is arbitrary. Our second main result provides combinatorial interpretations to the numbers $h_{i}^{+}(\Phi, m)$ similar to the ones given in [2] for $h_{i}(\Phi, m)$. To state this result we need to recall (or modify) some definitions and notation from [2]. For $y \in T_{m}$ consider the stabilizer of $y$ with respect to the $W$-action on $T_{m}$. This is a subgroup of $W$ generated by reflections. The minimum number of reflections needed to generate this subgroup is its rank and is denoted by $r(y)$. We may use the notation $r(x)$ for a $W$-orbit $x$ in $T_{m}$ since stabilizers of elements of $T_{m}$ in the same $W$-orbit are conjugate subgroups of $W$ and hence have the same rank. A subset $\mathcal{J}$ of $\Phi^{+}$is an order ideal if $\Phi^{+} \backslash \mathcal{J}$ is a filter. An increasing chain $\mathcal{J}_{1} \subseteq \mathcal{J}_{2} \subseteq \cdots \subseteq \mathcal{J}_{m}$ of ideals in $\Phi^{+}$is a geometric chain of ideals of length $m$ if

$$
\left(\mathcal{J}_{i}+\mathcal{J}_{j}\right) \cap \Phi^{+} \subseteq \mathcal{J}_{i+j}
$$

holds for all indices $i, j$ with $i+j \leq m$ and

$$
\left(\mathcal{I}_{i}+\mathcal{I}_{j}\right) \cap \Phi^{+} \subseteq \mathcal{I}_{i+j}
$$

holds for all indices $i, j$, where $\mathcal{I}_{i}=\Phi^{+} \backslash \mathcal{J}_{i}$ for $0 \leq i \leq m$ and $\mathcal{I}_{i}=\mathcal{I}_{m}$ for $i>m$. Such a chain is called positive if $\mathcal{J}_{m}$ contains the set of simple roots or, equivalently, if $\mathcal{I}_{m}$ is a positive filter. A positive root $\alpha$ is indecomposable of rank $m$ with respect to this increasing chain of ideals if $\alpha$ is a maximal element of $\mathcal{J}_{m} \backslash \mathcal{J}_{m-1}$ and it is not possible to write $\alpha=\beta+\gamma$ with $\beta \in \mathcal{J}_{i}$ and $\gamma \in \mathcal{J}_{j}$ for indices $i, j \geq 1$ with $i+j=m$. The following theorem refines part of Theorem 1.1.

Theorem 1.3. Let $\Phi$ be an irreducible crystallographic root system of rank $\ell$ with Weyl group $W, m$ be a positive integer and $O_{m}(\Phi)$ be the set of orbits of the action of $W$ on $\check{Q} /(m h-1) \grave{Q}$. For any $0 \leq i \leq \ell$ the following are equal:

(i) the number $h_{\ell-i}^{+}(\Phi, m)$,

(ii) the number of positive geometric chains of ideals in the root poset $\Phi^{+}$of length $m$ having $i$ indecomposable elements of rank $m$,

(iii) the number of orbits $x \in O_{m}(\Phi)$ with $r(x)=i$ and

(iv) the number of points in $\check{Q} \cap(m h-1) \overline{A_{\circ}}$ which lie in $i$ walls of $(m h-1) \overline{A_{\circ}}$. 
In particular, the number of positive geometric chains of ideals in $\Phi^{+}$of length $m$ is equal to $N^{+}(\Phi, m)$.

The equivalence of (iii) and (iv) follows essentially from the results of [10, Section 7.4]. In the special case $m=1$ the arrangement $\mathcal{A}_{\Phi}^{m}$ consists of the hyperplanes $H_{\alpha}$ and $H_{\alpha, 1}$ for all $\alpha \in \Phi$ and is known as the Catalan arrangement associated to $\Phi$, denoted $\mathrm{Cat}_{\Phi}$. Moreover a geometric chain of ideals consists of a single ideal $\mathcal{J}$ in $\Phi$. This chain is positive if $\mathcal{J}$ contains the set of simple roots or, equivalently, if $\mathcal{I}=\Phi^{+} \backslash \mathcal{J}$ is a positive filter and in that case the set of rank one indecomposable elements is the set of maximal elements of $\mathcal{J}$. We write $h_{i}^{+}(\Phi)$ instead of $h_{i}^{+}(\Phi, m)$ when $m=1$. Part of the next corollary is implicit in the work of E. Sommers [16, Section 6].

Corollary 1.4. Let $\Phi$ be an irreducible crystallographic root system of rank $\ell$ with Weyl group $W$ and $O(\Phi)$ be the set of orbits of the action of $W$ on $\check{Q} /(h-1) \grave{Q}$. For any $0 \leq i \leq \ell$ the following are equal:

(i) the number of ideals in the root poset $\Phi^{+}$which contain the set of simple roots and have $i$ maximal elements,

(ii) the number $h_{\ell-i}^{+}(\Phi)$ of bounded regions $R$ of $\mathrm{Cat}_{\Phi}$ in the fundamental chamber of $\mathcal{A}_{\Phi}$ such that $i$ walls of $R$ of the form $H_{\alpha, 1}$ do not separate $R$ from $A_{\circ}$,

(iii) the number of orbits $x \in O(\Phi)$ with $r(x)=i$,

(iv) the number of points in $\check{Q} \cap(h-1) \overline{A_{\circ}}$ which lie in $i$ walls of $(h-1) \overline{A_{\circ}}$ and

(v) the entry $h_{\ell-i}\left(\Delta_{+}(\Phi)\right)$ of the $h$-vector of the positive part of $\Delta(\Phi)$.

Our last theorem provides a different interpretation to the numbers $h_{i}^{+}(\Phi)$ in terms of order filters in $\Phi^{+}$.

Theorem 1.5. For any irreducible crystallographic root system $\Phi$ and any nonnegative integer $i$ the number $h_{i}^{+}(\Phi)$ is equal to the number of positive filters in $\Phi^{+}$having $i$ minimal elements.

Theorem 1.3 is proved in Sections 3 and 4 by means of two bijections. The first is the restriction of a bijection of [2, Section 3] and maps the set of positive geometric chains of ideals in $\Phi^{+}$of length $m$ to the set of bounded regions of $\mathcal{A}_{\Phi}^{m}$ in the fundamental chamber (Theorem 3.6) while the second maps this set of regions to the set of $W$-orbits of $T_{m}$. In the case $m=1$ the composite of these two bijections gives essentially a bijection of Sommers [16] from the set of positive filters in $\Phi^{+}$to $\breve{Q} \cap(h-1) \overline{A_{\circ}}$. The proof of Theorem 1.3 in these two sections parallels the one of Theorem 1.2 in [2] and for this reason most of the details are omitted. The main difference is that the unique alcove in a fixed bounded region of $\mathcal{A}_{\Phi}^{m}$ which is furthest away from $A_{\circ}$ plays the role played in [2] by the unique alcove in a region of $\mathcal{A}_{\Phi}^{m}$ closest to $A_{\circ}$. The existence of these maximal alcoves was first established and exploited in the special case $m=1$ by Sommers [16]. In Section 5 we prove Conjecture 1.2 when $m=1$ and when $\Phi$ has type $A, B$ or $C$ and $m$ is arbitrary (Corollary 5.5) using the fact that $h_{i}(\Phi, m)=h_{i}\left(\Delta^{m}(\Phi)\right)$ holds for all $i$ in these cases. A key ingredient in the proof is a new combinatorial interpretation (see part (iii) of Theorems 5.1 and 5.2) to the $f$-numbers defined from the $h_{i}(\Phi, m)$ and $h_{i}^{+}(\Phi, m)$ via the usual identity relating $f$-vectors and $h$-vectors of simplicial complexes. In Section 6 we compute the numbers which appear in Theorem 1.3 for root systems of classical type and those in Corollary 1.4 for root systems of exceptional type. We also prove Theorem 1.5 by exploiting the symmetry of the distribution 吾 Springer 
of the set of all filters in $\Phi^{+}$by the number of minimal elements, observed by D. Panyushev [13]. Some useful background material is summarized in Section 2. We conclude with some remarks in Section 7.

Apart from [2], our motivation for this work comes to a great extent from the papers by Fomin and Reading [7], Fomin and Zelevinsky [9] and Sommers [16].

\section{Preliminaries}

In this section we introduce notation and terminology and recall a few useful facts related to root systems, affine Weyl groups, generalized cluster complexes and the combinatorics of $\mathcal{A}_{\Phi}^{m}$. We refer to [11] and [2, 6, 7] for further background and references and warn the reader that, throughout the paper, some of our notation and terminology differs from that employed in [2] (this is done in part to ease the co-existence of order filters and order ideals in this paper, typically denoted by the letters $\mathcal{I}$ and $\mathcal{J}$, respectively, and in part to match some of the notation of [7]).

Root systems and Weyl groups. Let $V$ be an $\ell$-dimensional Euclidean space with inner product ( , ). Given a hyperplane arrangement $\mathcal{A}$ in $V$, meaning a discrete set of affine subspaces of $V$ of codimension one, the regions of $\mathcal{A}$ are the connected components of the space obtained from $V$ by removing the hyperplanes in $\mathcal{A}$. Let $\Phi$ be a crystallographic root system spanning $V$. For any real $k$ and $\alpha \in \Phi$ we denote by $H_{\alpha, k}$ the hyperplane in $V$ defined by the equation $(\alpha, x)=k$ and set $H_{\alpha}=H_{\alpha, 0}$. We fix a positive system $\Phi^{+} \subseteq \Phi$ and the corresponding (ordered) set of simple roots $\Pi=\left\{\sigma_{1}, \ldots, \sigma_{\ell}\right\}$. For $1 \leq i \leq \ell$ we denote by $s_{i}$ the orthogonal reflection in the hyperpane $H_{\sigma_{i}}$, called a simple reflection. We will often write $\Phi_{I}$ instead of $\Phi$, where $I$ is an index set in bijection with $\Pi$, and denote by $\Phi_{J}$ the parabolic root system corresponding to $J \subseteq I$. If $\Phi$ is irreducible we denote by $\tilde{\alpha}$ the highest positive root, by $e_{1}, e_{2}, \ldots, e_{\ell}$ the exponents and by $h$ the Coxeter number of $\Phi$ and set $p=m h-1$, where $m$ is a fixed positive integer. The following well known lemmas will be used, as in [2].

Lemma 2.1. ([2, Lemma 2.1]) (i) If $\alpha_{1}, \alpha_{2}, \ldots, \alpha_{r} \in \Phi^{+}$with $r \geq 2$ and $\alpha=\alpha_{1}+$ $\alpha_{2}+\cdots+\alpha_{r} \in \Phi^{+}$then there exists $i$ with $1 \leq i \leq r$ such that $\alpha-\alpha_{i} \in \Phi^{+}$.

(ii) (cf. [13, 16]) If $\alpha_{1}, \alpha_{2}, \ldots, \alpha_{r} \in \Phi$ and $\alpha_{1}+\alpha_{2}+\cdots+\alpha_{r}=\alpha \in \Phi$ then $\alpha_{1}=\alpha$ or there exists $i$ with $2 \leq i \leq r$ such that $\alpha_{1}+\alpha_{i} \in \Phi \cup\{0\}$.

Lemma 2.2. ([4, Ch. 6, 1.11, Proposition 31] [11, p. 84]) If $\Phi$ is irreducible and $\tilde{\alpha}=$ $\sum_{i=1}^{\ell} c_{i} \sigma_{i}$ then $\sum_{i=1}^{\ell} c_{i}=h-1$.

We denote by $\mathcal{A}_{\Phi}$ the Coxeter arrangement associated to $\Phi$, i.e. the collection of linear hyperplanes $H_{\alpha}$ in $V$ with $\alpha \in \Phi$, and by $W$ the corresponding Weyl group, generated by the reflections in these hyperplanes. Thus $W$ is finite and minimally generated by the set of simple reflections, it leaves $\Phi$ invariant and acts simply transitively on the set of regions of $\mathcal{A}_{\Phi}$, called chambers. The fundamental chamber is the region defined by the inequalities $0<(\alpha, x)$ for $\alpha \in \Phi^{+}$. A subset of $V$ is called dominant if it is contained in the fundamental chamber. The coroot lattice $\check{Q}$ of $\Phi$ is the $\mathbb{Z}$-span of the set of coroots

$$
\Phi^{\vee}=\left\{\frac{2 \alpha}{(\alpha, \alpha)}: \alpha \in \Phi\right\} .
$$


From now on we assume for simplicity that $\Phi$ is irreducible. The group $W$ acts on the lattice $\check{Q}$ and on its sublattice $p \check{Q}$, hence it also acts on the quotient $T_{m}(\Phi)=\check{Q} / p \check{Q}$. We denote by $O_{m}(\Phi)$ the set of orbits of the $W$-action on $T_{m}(\Phi)$ and use the notation $T(\Phi)$ and $O(\Phi)$ when $m=1$. We denote by $\widetilde{\mathcal{A}}_{\Phi}$ the affine Coxeter arrangement, which is the infinite hyperplane arrangement in $V$ consisting of the hyperplanes $H_{\alpha, k}$ for $\alpha \in \Phi$ and $k \in \mathbb{Z}$, and by $W_{a}$ the affine Weyl group, generated by the reflections in the hyperplanes of $\widetilde{\mathcal{A}}_{\Phi}$. The group $W_{a}$ is the semidirect product of $W$ and the translation group in $V$ corresponding to the coroot lattice $\breve{Q}$ and is minimally generated by the set $\left\{s_{0}, s_{1}, \ldots, s_{\ell}\right\}$ of simple affine reflections, where $s_{0}$ is the reflection in the hyperplane $H_{\widetilde{\alpha}, 1}$. For $w \in W_{a}$ and $0 \leq i \leq \ell$, the reflection $s_{i}$ is a right ascent of $w$ if $\ell\left(w s_{i}\right)>\ell(w)$, where $\ell(w)$ is the length of the shortest expression of $w$ as a product of simple affine reflections. The group $W_{a}$ acts simply transitively on the set of regions of $\widetilde{\mathcal{A}}_{\Phi}$, called alcoves. The fundamental alcove of $\widetilde{\mathcal{A}}_{\Phi}$ can be defined as

$$
A_{\circ}=\left\{x \in V: 0<\left(\sigma_{i}, x\right) \text { for } 1 \leq i \leq \ell \text { and }(\tilde{\alpha}, x)<1\right\} .
$$

Note that every alcove can be written as $w A_{\circ}$ for a unique $w \in W_{a}$. Moreover, given $\alpha \in \Phi^{+}$, there exists a unique integer $r$, denoted $r(w, \alpha)$, such that $r-1<(\alpha, x)<r$ holds for all $x \in w A_{\circ}$. The next lemma is a reformulation of the main result of [15].

Lemma 2.3. ([15, Theorem 5.2]). Let $r_{\alpha}$ be an integer for each $\alpha \in \Phi^{+}$. There exists $w \in W_{a}$ such that $r(w, \alpha)=r_{\alpha}$ for each $\alpha \in \Phi^{+}$if and only if

$$
r_{\alpha}+r_{\beta}-1 \leq r_{\alpha+\beta} \leq r_{\alpha}+r_{\beta}
$$

for all $\alpha, \beta \in \Phi^{+}$with $\alpha+\beta \in \Phi^{+}$.

We say that two open regions in $V$ are separated by a hyperplane $H \in \widetilde{\mathcal{A}}_{\Phi}$ if they lie in different half-spaces relative to $H$. If $R$ is a region of a subarrangement of $\widetilde{\mathcal{A}}_{\Phi}$ or the closure of such a region (in particular, if $R$ is a chamber or an alcove), we refer to the hyperplanes of $\widetilde{\mathcal{A}}_{\Phi}$ which support facets of the closure of $R$ as the walls of $R$.

Generalized cluster complexes. Let $\Phi$ be crystallographic (possibly reducible) of rank $\ell$. The generalized cluster complex $\Delta^{m}(\Phi)$ is an abstract simplicial complex on the vertex set $\Phi_{\geq-1}^{m}$ consisting of the negative simple roots and $m$ copies of each positive root; we refer to [7, Section 1.2] for the definition. It is a pure complex of dimension $\ell-1$ [7, Proposition 1.7]. If $\Phi$ is a direct product $\Phi=\Phi_{1} \times \Phi_{2}$ then $\Delta^{m}(\Phi)$ is the simplicial join of $\Delta^{m}\left(\Phi_{1}\right)$ and $\Delta^{m}\left(\Phi_{2}\right)$. We denote by $\Delta_{+}^{m}(\Phi)$ the induced subcomplex of $\Delta^{m}(\Phi)$ on the set of vertices obtained from $\Phi_{\geq-1}^{m}$ by removing the negative simple roots and call this simplicial complex the positive part of $\Delta^{m}(\Phi)$. For $0 \leq i \leq \ell$ we denote by $f_{i-1}\left(\Delta^{m}(\Phi)\right)$ and $f_{i-1}\left(\Delta_{+}^{m}(\Phi)\right)$ the number of $(i-1)$-dimensional faces of the complex $\Delta^{m}(\Phi)$ and $\Delta_{+}^{m}(\Phi)$, respectively. These numbers are related to the $h_{i}\left(\Delta^{m}(\Phi)\right)$ and $h_{i}\left(\Delta_{+}^{m}(\Phi)\right)$ by the equations

$$
\sum_{i=0}^{\ell} f_{i-1}\left(\Delta^{m}(\Phi)\right)(x-1)^{\ell-i}=\sum_{i=0}^{\ell} h_{i}\left(\Delta^{m}(\Phi)\right) x^{\ell-i}
$$

and

$$
\sum_{i=0}^{\ell} f_{i-1}\left(\Delta_{+}^{m}(\Phi)\right)(x-1)^{\ell-i}=\sum_{i=0}^{\ell} h_{i}\left(\Delta_{+}^{m}(\Phi)\right) x^{\ell-i}
$$

respectively.

은 Springer 
Following [7,18] we give explicit combinatorial descriptions of the complexes $\Delta^{m}(\Phi)$ and $\Delta_{+}^{m}(\Phi)$ when $\Phi$ has type $A, B$ or $C$. For $\Phi=A_{n-1}$ let $\mathbf{P}$ be a convex polygon with $m n+2$ vertices. A diagonal of $\mathbf{P}$ is called $m$-allowable if it divides $\mathbf{P}$ into two polygons each with number of vertices congruent to $2 \bmod m$. Vertices of $\Delta^{m}(\Phi)$ are the $m$-allowable diagonals of $\mathbf{P}$ and faces are the sets of pairwise noncrossing diagonals of this kind. For $\Phi=B_{n}$ or $C_{n}$ let $\mathbf{Q}$ be a centrally symmetric convex polygon with $2 m n+2$ vertices. A vertex of $\Delta^{m}(\Phi)$ is either a diameter of $\mathbf{Q}$, i.e. a diagonal connecting antipodal vertices, or a pair of $m$-allowable diagonals related by a half-turn about the center of $\mathbf{Q}$. A set of vertices of $\Delta^{m}(\Phi)$ forms a face if the diagonals of $\mathbf{Q}$ defining these vertices are pairwise noncrossing. In all cases the explicit bijection of $\Phi_{\geq-1}^{m}$ with the set of allowable diagonals of $\mathbf{P}$ or $\mathbf{Q}$ just described is analogous to the one given in [9, Section 3.5] for the usual cluster complex $\Delta(\Phi)$, so that the negative simple roots form an $m$-snake of allowable diagonals in $\mathbf{P}$ or $\mathbf{Q}$ and $\Delta_{+}^{m}(\Phi)$ is the subcomplex of $\Delta^{m}(\Phi)$ obtained by removing the vertices in the $m$-snake.

The negative part of a face $c$ of $\Delta^{m}(\Phi)$ is the set of indices $J \subseteq I$, where $\Phi=\Phi_{I}$, which correspond to the negative simple roots contained in $c$. The next lemma appears as [9, Proposition 3.6] in the case $m=1$ and follows from the explicit description of the relevant complexes in the remaining cases.

Lemma 2.4. Assume that either $m=1$ or $\Phi_{I}$ has type $A, B$ or $C$. For any $J \subseteq I$ the map $c \mapsto c \backslash\left\{-\alpha_{i}: i \in J\right\}$ is a bijection from the set of faces of $\Delta^{m}\left(\Phi_{I}\right)$ with negative part $J$ to the set of faces of $\Delta_{+}^{m}\left(\Phi_{I \backslash J}\right)$. In particular

$$
f_{k-1}\left(\Delta^{m}\left(\Phi_{I}\right)\right)=\sum_{J \subseteq I} f_{k-|J|-1}\left(\Delta_{+}^{m}\left(\Phi_{I \backslash J}\right)\right),
$$

where $f_{i-1}(\Delta)=0$ if $i<0$ for any complex $\Delta$.

For $m=1$ essentially the same equation as (6) has appeared in the context of quiver representations in [12, Section 6].

Regions of $\mathcal{A}_{\Phi}^{m}$ and chains of filters. Let $\Phi$ be irreducible and crystallographic of rank $\ell$. For $0 \leq i \leq \ell$ let $h_{i}(\Phi, m)$ be the number of dominant regions $R$ of $\mathcal{A}_{\Phi}^{m}$ for which exactly $\ell-i$ walls of $R$ of the form $H_{\alpha, m}$ separate $R$ from $A_{\circ}$, as in Section 1. We recall another combinatorial interpretation of $h_{i}(\Phi, m)$ from [2] using slightly different terminology. We call a decreasing chain

$$
\Phi^{+}=\mathcal{I}_{0} \supseteq \mathcal{I}_{1} \supseteq \mathcal{I}_{2} \supseteq \cdots \supseteq \mathcal{I}_{m}
$$

of filters in $\Phi^{+}$a geometric chain of filters of length $m$ if (1) and (2) hold under the same conventions as in Section 1 (the term co-filtered chain of dual order ideals was used in [2] instead). A positive root $\alpha$ is indecomposable of rank $m$ with respect to this chain if $\alpha \in \mathcal{I}_{m}$ and it is not possible to write $\alpha=\beta+\gamma$ with $\beta \in \mathcal{I}_{i}$ and $\gamma \in \mathcal{I}_{j}$ for indices $i, j \geq 0$ with $i+j=m$. Let $R_{\mathcal{I}}$ be the set of points $x \in V$ which satisfy

$$
\begin{array}{ll}
(\alpha, x)>r, & \text { if } \alpha \in \mathcal{I}_{r} \\
0<(\alpha, x)<r, & \text { if } \alpha \in \mathcal{J}_{r}
\end{array}
$$

for $0 \leq r \leq m$, where $\mathcal{J}_{r}=\Phi^{+} \backslash \mathcal{I}_{r}$. The following statement combines parts of Theorems 3.6 and 3.11 in [2]. 
Theorem 2.5. The map $\mathcal{I} \mapsto R_{\mathcal{I}}$ is a bijection from the set of geometric chains of filters of length $m$ in $\Phi^{+}$to the set of dominant regions of $\mathcal{A}_{\Phi}^{m}$. Moreover a positive root $\alpha$ is indecomposable of rank $m$ with respect to $\mathcal{I}$ if and only if $H_{\alpha, m}$ is a wall of $R_{\mathcal{I}}$ which separates $R_{\mathcal{I}}$ from $A_{\circ}$.

In particular $h_{i}(\Phi, m)$ is equal to the number of geometric chains of filters in $\Phi^{+}$of length $m$ having $\ell-i$ indecomposable elements of rank $m$.

By modifying the definition given earlier or using the interpretation in the last statement of the previous theorem we can define the numbers $h_{i}(\Phi, m)$ when $\Phi$ is reducible as well. Clearly

$$
h_{k}\left(\Phi_{1} \times \Phi_{2}, m\right)=\sum_{i+j=k} h_{i}\left(\Phi_{1}, m\right) h_{j}\left(\Phi_{2}, m\right)
$$

for any crystallographic root systems $\Phi_{1}, \Phi_{2}$.

\section{Chains of ideals, bounded regions and maximal alcoves}

In this section we generalize some of the results of Sommers [16] on bounded dominant regions of $\mathrm{Cat}_{\Phi}$ and positive filters in $\Phi^{+}$to bounded dominant regions of $\mathcal{A}_{\Phi}^{m}$ and positive geometric chains of ideals and establish the equality of the numbers appearing in (i) and (ii) in the statement of Theorem 1.3. The results of this and the following section are analogues of the results of Sections 3 and 4 of [2] on the set of all dominant regions of $\mathcal{A}_{\Phi}^{m}$. Their proofs are obtained by minor adjustments from those of [2], suggested by the modifications of the relevant definitions, and thus are only sketched or omitted.

Let $\Phi$ be irreducible and crystallographic of rank $\ell$ and let $\mathcal{J}$ be a positive geometric chain of ideals

$$
\emptyset=\mathcal{J}_{0} \subseteq \mathcal{J}_{1} \subseteq \mathcal{J}_{2} \subseteq \cdots \subseteq \mathcal{J}_{m}
$$

in $\Phi^{+}$of length $m$, so that (1) and (2) hold, where $\mathcal{I}_{i}=\Phi^{+} \backslash \mathcal{J}_{i}$, and $\Pi \subseteq \mathcal{J}_{m}$. We define

$$
r_{\alpha}(\mathcal{J})=\min \left\{r_{1}+r_{2}+\cdots+r_{k}: \alpha=\alpha_{1}+\alpha_{2}+\cdots+\alpha_{k} \text { with } \alpha_{i} \in \mathcal{J}_{r_{i}} \text { for all } i\right\}
$$

for any $\alpha \in \Phi^{+}$. Observe that $r_{\alpha}(\mathcal{J})$ is well defined since $\Pi \subseteq \mathcal{J}_{m}$ and that $r_{\alpha}(\mathcal{J}) \leq r$ for $\alpha \in \mathcal{J}_{r}$, with $r_{\alpha}(\mathcal{J})=1$ if and only if $\alpha \in \mathcal{J}_{1}$.

Lemma 3.1. If $\alpha=\alpha_{1}+\alpha_{2}+\cdots+\alpha_{k} \in \Phi^{+}$and $\alpha_{i} \in \Phi^{+}$for all $i$ then

$$
r_{\alpha}(\mathcal{J}) \leq \sum_{i=1}^{k} r_{\alpha_{i}}(\mathcal{J})
$$

Proof: This is clear from the definition.

Lemma 3.2. Let $\alpha \in \Phi^{+}$and $r_{\alpha}(\mathcal{J})=r$.

(i) If $r \leq m$ then $\alpha \in \mathcal{J}_{r}$.

(ii) If $r>m$ then there exist $\beta, \gamma \in \Phi^{+}$with $\alpha=\beta+\gamma$ and $r=r_{\beta}(\mathcal{J})+r_{\gamma}(\mathcal{J})$. Moreover we may choose $\beta$ so that $r_{\beta}(\mathcal{J}) \leq m$.

은 Springer 
Proof: Analogous to the proof of [2, Lemma 3.2].

Lemma 3.3. If $\alpha, \beta, \alpha+\beta \in \Phi^{+}$and $a, b$ are integers such that $r_{\alpha+\beta}(\mathcal{J}) \leq a+b$ then $r_{\alpha}(\mathcal{J}) \leq a$ or $r_{\beta}(\mathcal{J}) \leq b$.

Proof: By induction on $r_{\alpha+\beta}(\mathcal{J})$, as in the proof of [2, Lemma 3.3].

Corollary 3.4. We have

$$
r_{\alpha}(\mathcal{J})+r_{\beta}(\mathcal{J})-1 \leq r_{\alpha+\beta}(\mathcal{J}) \leq r_{\alpha}(\mathcal{J})+r_{\beta}(\mathcal{J})
$$

whenever $\alpha, \beta, \alpha+\beta \in \Phi^{+}$.

Proof: The second inequality is a special case of Lemma 3.1 and the first follows from Lemma 3.3 letting $a=r_{\alpha}(\mathcal{J})-1$ and $b=r_{\alpha+\beta}(\mathcal{J})-a$.

We denote by $R^{\mathcal{J}}$ the set of points $x \in V$ which satisfy the inequalities in (7). Since $\Pi \subseteq \mathcal{J}_{m}$ we have $0<\left(\sigma_{i}, x\right)<m$ for all $1 \leq i \leq \ell$ and $x \in R^{\mathcal{J}}$ and therefore $R^{\mathcal{J}}$ is bounded.

Proposition 3.5. There exists a unique $w \in W_{a}$ such that $r(w, \alpha)=r_{\alpha}(\mathcal{J})$ for $\alpha \in \Phi^{+}$. Moreover, $w A_{\circ} \subseteq R^{\mathcal{J}}$. In particular, $R^{\mathcal{J}}$ is nonempty.

Proof: The existence in the first statement follows from Lemma 2.3 and Corollary 3.4 while uniqueness is obvious. For the second statement let $\alpha \in \Phi^{+}$and $1 \leq r \leq m$. Part (i) of Lemma 3.2 implies that $r_{\alpha}(\mathcal{J}) \leq r$ if and only if $\alpha \in J_{r}$. Hence from the inequalities

$$
r_{\alpha}(\mathcal{J})-1<(\alpha, x)<r_{\alpha}(\mathcal{J})
$$

which hold for $x \in w A_{\circ}$, we conclude that $w A_{\circ} \subseteq R^{\mathcal{J}}$.

Let $\psi$ be the map which assigns the set $R^{\mathcal{J}}$ to a positive geometric chain of ideals $\mathcal{J}$ in $\Phi^{+}$ of length $m$. Conversely, given a bounded dominant region $R$ of $\mathcal{A}_{\Phi}^{m}$ let $\phi(R)$ be the sequence $\emptyset=\mathcal{J}_{0} \subseteq \mathcal{J}_{1} \subseteq \mathcal{J}_{2} \subseteq \cdots \subseteq \mathcal{J}_{m}$ where $\mathcal{J}_{r}$ is the set of $\alpha \in \Phi^{+}$for which $(\alpha, x)<r$ holds in $R$. Clearly each $\mathcal{J}_{r}$ is an ideal in $\Phi^{+}$.

Theorem 3.6. The map $\psi$ is a bijection from the set of positive geometric chains of ideals in $\Phi^{+}$of length $m$ to the set of bounded dominant regions of $\mathcal{A}_{\Phi}^{m}$, and the map $\phi$ is its inverse.

Proof: That $\psi$ is well defined follows from Proposition 3.5, which guarantees that $R^{\mathcal{J}}$ is nonempty (and bounded). To check that $\phi$ is well defined observe that if $R$ is a bounded dominant region of $\mathcal{A}_{\Phi}^{m}$ and if $(\alpha, x)<i$ and $(\beta, x)<j$ hold for $x \in R$ then $(\alpha+\beta, x)<i+j$ must hold for $x \in R$, so that $\phi(\mathcal{J})$ satisfies (1). Similarly, $\phi(\mathcal{J})$ satisfies (2). That $\Pi \subseteq \mathcal{J}_{m}$ follows from [1, Lemma 4.1]. It is clear that $\psi$ and $\phi$ are inverses of each other.

Let $R=R^{\mathcal{J}}$ be a bounded dominant region of $\mathcal{A}_{\Phi}^{m}$, where $\mathcal{J}=\phi(R)$. Let $w_{R}$ denote the element of the affine Weyl group $W_{a}$ which is assigned to $\mathcal{J}$ in Proposition 3.5. The 
following proposition implies that $w_{R} A_{\circ}$ is the alcove in $R$ which is the furthest away from $A_{\circ}$. In the special case $m=1$ the existence of such an alcove was established by Sommers [16, Proposition 5.4].

Proposition 3.7. Let $R$ be a bounded dominant region of $\mathcal{A}_{\Phi}^{m}$. The element $w_{R}$ is the unique $w \in W_{a}$ such that $w A_{\circ} \subseteq R$ and whenever $\alpha \in \Phi^{+}, r \in \mathbb{Z}$ and $(\alpha, x)>r$ holds for some $x \in R$ we have $(\alpha, x)>r$ for all $x \in w A_{\circ}$.

Proof: Analogous to the proof of [2, Proposition 3.7].

We now introduce the notion of an indecomposable element with respect to the increasing chain of ideals $\mathcal{J}$.

Definition 3.8. Given $1 \leq r \leq m$, a root $\alpha \in \Phi^{+}$is indecomposable of rank $r$ with respect to $\mathcal{J}$ if $\alpha \in \mathcal{J}_{r}$ and

(i) $r_{\alpha}(\mathcal{J})=r$,

(ii) it is not possible to write $\alpha=\beta+\gamma$ with $\beta \in \mathcal{J}_{i}$ and $\gamma \in \mathcal{J}_{j}$ for indices $i, j \geq 1$ with $i+j=r$ and

(iii) if $r_{\alpha+\beta}(\mathcal{J})=t \leq m$ for some $\beta \in \Phi^{+}$then $\beta \in \mathcal{J}_{t-r}$.

Observe that, by part (i) of Lemma 3.2, the assumption $\alpha \in \mathcal{J}_{r}$ in this definition is actually implied by condition (i). For $r=m$ the definition is equivalent to the one proposed in Section 1 , as the following lemma shows.

Lemma 3.9. A positive root $\alpha$ is indecomposable of rank $m$ with respect to $\mathcal{J}$ if and only if $\alpha$ is a maximal element of $\mathcal{J}_{m} \backslash \mathcal{J}_{m-1}$ and it is not possible to write $\alpha=\beta+\gamma$ with $\beta \in \mathcal{J}_{i}$ and $\gamma \in \mathcal{J}_{j}$ for indices $i, j \geq 1$ with $i+j=m$.

Proof: Suppose that $\alpha \in \mathcal{J}_{m}$ is indecomposable of rank $m$. Since $r_{\alpha}(\mathcal{J})=m$ we must have $\alpha \notin \mathcal{J}_{m-1}$. Hence to show that $\alpha$ satisfies the condition in the statement of the lemma it suffices to show that $\alpha$ is maximal in $\mathcal{J}_{m}$. If not then by Lemma 2.1 (i) there exists $\beta \in \Phi^{+}$ such that $\alpha+\beta \in \mathcal{J}_{m}$. Then clearly $r_{\alpha+\beta}(\mathcal{J}) \leq m$ and $r_{\alpha+\beta}(\mathcal{J}) \geq m$ by Corollary 3.4. Hence $r_{\alpha+\beta}(\mathcal{J})=m$ and condition (iii) of Definition 3.8 leads to a contradiction.

For the converse, suppose that $\alpha \in \mathcal{J}_{m}$ satisfies the condition in the statement of the lemma. In view of part (i) of Lemma 3.2, condition (iii) in Definition 3.8 is satisfied since $\alpha$ is assumed to be maximal in $\mathcal{J}_{m}$. Hence to show that $\alpha$ is indecomposable of rank $m$ it suffices to show that $r_{\alpha}(\mathcal{J})=m$. This is implied by the assumption that $\alpha \notin \mathcal{J}_{m-1}$ and part (i) of Lemma 3.2.

Lemma 3.10. Suppose that $\alpha$ is indecomposable with respect to $\mathcal{J}$.

(i) We have $r_{\alpha}(\mathcal{J})=r_{\beta}(\mathcal{J})+r_{\gamma}(\mathcal{J})-1$ whenever $\alpha=\beta+\gamma$ with $\beta, \gamma \in \Phi^{+}$.

(ii) We have $r_{\alpha}(\mathcal{J})+r_{\beta}(\mathcal{J})=r_{\alpha+\beta}(\mathcal{J})$ whenever $\beta, \alpha+\beta \in \Phi^{+}$.

Proof: Analogous to the proof of [2, Lemma 3.10]. For part (ii), letting $r_{\alpha}(\mathcal{J})=$ $r$ and $r_{\alpha+\beta}(\mathcal{J})=t$, we prove instead that $r_{\beta}(\mathcal{J}) \leq t-r$. This implies the result by Corollary 3.4. 


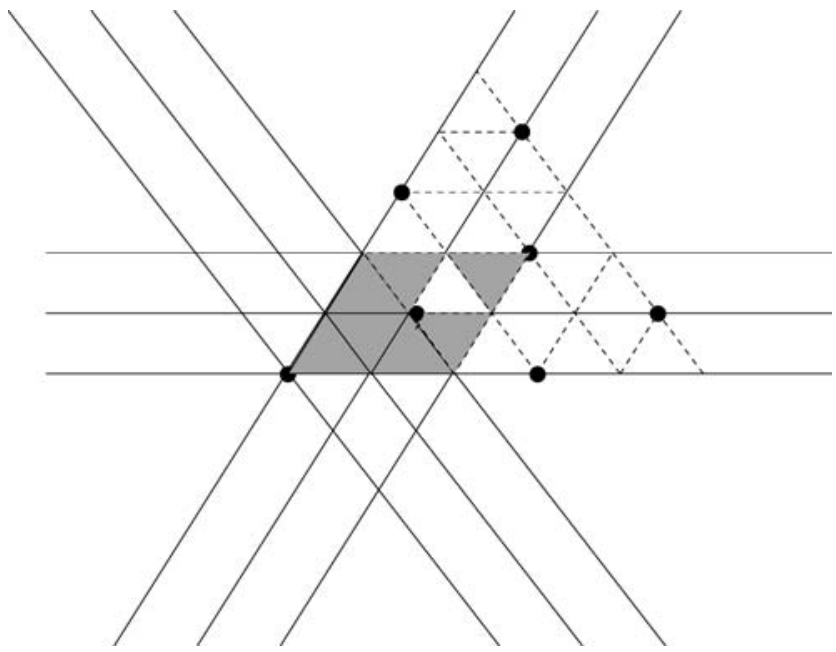

Fig. 1 The maximal alcoves of the bounded dominant regions and the simplex $p \overline{A_{\circ}}$ for $\Phi=A_{2}$ and $m=2$.

The following theorem explains the connection between indecomposable elements of $\mathcal{J}$ and walls of $R^{\mathcal{J}}$.

Theorem 3.11. If $\mathcal{J}$ is a positive geometric chain of ideals in $\Phi^{+}$of length $m$ with corresponding region $R=R^{\mathcal{J}}$ and $1 \leq r \leq m$ then the following sets are equal:

(i) the set of indecomposable roots $\alpha \in \Phi^{+}$with respect to $\mathcal{J}$ of rank $r$,

(ii) the set of $\alpha \in \Phi^{+}$such that $H_{\alpha, r}$ is a wall of $R$ which does not separate $R$ from $A_{\circ}$ and

(iii) the set of $\alpha \in \Phi^{+}$such that $H_{\alpha, r}$ is a wall of $w_{R} A_{\circ}$ which does not separate $w_{R} A_{\circ}$ from $A_{\circ}$.

Proof: We prove that $F_{r}(R) \subseteq F_{r}(\mathcal{J}) \subseteq F_{r}\left(w_{R}\right) \subseteq F_{r}(R)$ for the three sets defined in the statement of the theorem as in the proof of [2, Theorem 3.11], replacing the inequalities $(\alpha, x)>k$ which appear there by $(\alpha, x)<r$ and recalling from the proof of Proposition 3.7 that $(\alpha, x)<r_{\alpha}(\mathcal{J})$ holds for all $\alpha \in \Phi^{+}$and $x \in R^{\mathcal{J}}$.

We denote by $W_{m}(\Phi)$ the subset of $W_{a}$ consisting of the elements $w_{R}$ for the bounded dominant regions $R$ of $\mathcal{A}_{\Phi}^{m}$; see Figure 1 for the case $\Phi=A_{2}$ and $m=2$. We abbreviate this set as $W(\Phi)$ in the case $m=1$. The elements of $W(\Phi)$ are called maximal in [16].

Corollary 3.12. For any nonnegative integers $i_{1}, i_{2}, \ldots, i_{m}$ the following are equal:

(i) the number of positive geometric chains of ideals in $\Phi^{+}$of length $m$ having $i_{r}$ indecomposable elements of rank $r$ for each $1 \leq r \leq m$,

(ii) the number of bounded dominant regions $R$ of $\mathcal{A}_{\Phi}^{m}$ such that $i_{r}$ walls of $R$ of the form $H_{\alpha, r}$ do not separate $R$ from $A_{\circ}$ for each $1 \leq r \leq m$ and

(iii) the number of $w \in W_{m}(\Phi)$ such that $i_{r}$ walls of $w A_{\circ}$ of the form $H_{\alpha, r}$ do not separate $w A_{\circ}$ from $A_{\circ}$ for each $1 \leq r \leq m$. 
Proof: Combine Theorems 3.6 and 3.11 .

The following corollary is immediate.

Corollary 3.13. For any nonnegative integer $i$ the numbers which appear in (i) and (ii) in the statement of Theorem 1.3 are both equal to the number of $w \in W_{m}(\Phi)$ such that $i$ walls of $w A_{\circ}$ of the form $H_{\alpha, m}$ do not separate $w A_{\circ}$ from $A_{\circ}$.

As was the case with $h_{i}(\Phi, m)$, the interpretation in part (ii) of Theorem 1.3 mentioned in the previous corollary or the original definition can be used to define $h_{i}^{+}(\Phi, m)$ when $\Phi$ is reducible. Equivalently we define

$$
h_{k}^{+}\left(\Phi_{1} \times \Phi_{2}, m\right)=\sum_{i+j=k} h_{i}^{+}\left(\Phi_{1}, m\right) h_{j}^{+}\left(\Phi_{2}, m\right)
$$

for any crystallographic root systems $\Phi_{1}, \Phi_{2}$. We now consider the special case $m=1$. A positive geometric chain of ideals $\mathcal{J}$ of length $m$ in this case is simply a single ideal $\mathcal{J}$ in $\Phi^{+}$such that $\Pi \subseteq \mathcal{J}$, meaning that $\mathcal{I}=\Phi^{+} \backslash \mathcal{J}$ is a positive filter. By Lemma 3.9 the rank one indecomposable elements of $\mathcal{J}$ are exactly the maximal elements of $\mathcal{J}$.

Corollary 3.14. For any nonnegative integer $i$ the following are equal to $h_{\ell-i}^{+}(\Phi)$ :

(i) the number of ideals in the root poset $\Phi^{+}$which contain all simple roots and have $i$ maximal elements,

(ii) the number of bounded dominant regions $R$ of $\mathrm{Cat}_{\Phi}$ such that $i$ walls of $R$ of the form $H_{\alpha, 1}$ do not separate $R$ from $A_{\circ}$,

(iii) the number of $w \in W(\Phi)$ such that $i$ walls of $w A_{\circ}$ of the form $H_{\alpha, 1}$ do not separate $w A_{\circ}$ from $A_{\circ}$ and

(iv) the number of elements $w \in W(\Phi)$ having $i$ right ascents.

Proof: This follows from the case $m=1$ of Corollary 3.12 and [2, Lemma 2.5].

\section{Coroot lattice points and the affine Weyl group}

In this section we complete the proof of Theorem 1.3 (see Corollary 4.4). We assume that $\Phi$ is irreducible and crystallographic of rank $\ell$.

As in [2, Section 4], by the reflection in $W$ corresponding to a hyperplane $H_{\alpha, k}$ we mean the reflection in the linear hyperplane $H_{\alpha}$. We let $p=m h-1$, as in Section 2, and $D_{m}(\Phi)=\check{Q} \cap p \overline{A_{\circ}}$. The following elementary lemma, for which a detailed proof can be found in [10, Section 7.4], implies that $D_{m}(\Phi)$ is a set of representatives for the orbits of the $W$-action on $T_{m}(\Phi)$.

Lemma 4.1. (cf. [10, Lemma 7.4.1]) The natural inclusion map from $D_{m}(\Phi)$ to the set $O_{m}(\Phi)$ of orbits of the $W$-action on $T_{m}(\Phi)$ is a bijection.

Moreover, if $y \in D_{m}(\Phi)$ then the stabilizer of $y$ with respect to the $W$-action on $T_{m}(\Phi)$ is the subgroup of $W$ generated by the reflections corresponding to the walls of $p \overline{A_{\circ}}$ which contain $y$. In particular, $r(y)$ is equal to the number of walls of $p \overline{A_{\circ}}$ which contain $y$. 
We will define a bijection $\rho: W_{m}(\Phi) \rightarrow D_{m}(\Phi)$ such that for $w \in W_{m}(\Phi)$, the number of walls of $w A_{\circ}$ of the form $H_{\alpha, m}$ which do not separate $w A_{\circ}$ from $A_{\circ}$ is equal to the number of walls of $p \overline{A_{\circ}}$ which contain $\rho(w)$. Let $R_{f}$ be the region of $\mathcal{A}_{\Phi}^{m}$ defined by the inequalities $m-1<(\alpha, x)<m$ for $1 \leq i \leq \ell$. Let $w_{f}=w_{R_{f}}$ be the unique element $w$ of $W_{m}(\Phi)$ such that $w A_{\circ} \subseteq R_{f}$. We define the map $\rho: W_{m}(\Phi) \rightarrow \check{Q}$ by

$$
\rho(w)=\left(w_{f} w^{-1}\right) \cdot 0
$$

for $w \in W_{m}(\Phi)$. Observe that, by Lemma 2.2, the alcove $w_{f} A_{\circ}$ can be described explicitly as the open simplex in $V$ defined by the linear inequalities $\left(\sigma_{i}, x\right)<m$ for $1 \leq i \leq \ell$ and $(\tilde{\alpha}, x)>m h-m-1$. For any $1 \leq r \leq m$ we define the simplex

$$
\Sigma_{m}^{r}=\left\{x \in V: m-r \leq\left(\sigma_{i}, x\right) \text { for } 1 \leq i \leq \ell \text { and }(\tilde{\alpha}, x) \leq m h-m+r-1\right\},
$$

so that $\Sigma_{m}^{m}=p \overline{A_{\circ}}$. For any $\ell$-dimensional simplex $\Sigma$ in $V$ bounded by hyperplanes $H_{\alpha, k}$ in $\widetilde{\mathcal{A}_{\Phi}}$ with $\alpha \in \Pi \cup\{\tilde{\alpha}\}$ we denote by $H(\Sigma, i)$ the wall of $\Sigma$ orthogonal to $\tilde{\alpha}$ or $\sigma_{i}$, if $i=0$ or $i>0$, respectively. We write $H(w, i)$ instead of $H\left(w \overline{A_{\circ}}, i\right)$ for $w \in W_{a}$. The reader is invited to test the results that follow in the case pictured in Figure 1.

Theorem 4.2. The map $\rho$ is a bijection from $W_{m}(\Phi)$ to $D_{m}(\Phi)$. Moreover for any $w \in$ $W_{m}(\Phi), 1 \leq r \leq m$ and $0 \leq i \leq \ell$, the point $\rho(w)$ lies on the wall $H\left(\Sigma_{m}^{r}, i\right)$ if and only if the wall $\left(w w_{f}^{-1}\right) H\left(w_{f}, i\right)$ of $w A_{\circ}$ is of the form $H_{\alpha, r}$ and does not separate $w A_{\circ}$ from $A_{\circ}$.

Proof: Analogous to the proof of [2, Theorem 4.2].

Corollary 4.3. For any nonnegative integers $i_{1}, i_{2}, \ldots, i_{m}$ each of the quantities which appear in the statement of Corollary 3.12 is equal to the number of points in $D_{m}(\Phi)$ which lie in $i_{r}$ walls of $\Sigma_{m}^{r}$ for all $1 \leq r \leq m$.

Proof: This follows from Theorem 4.2.

The next corollary completes the proof of Theorem 1.3.

Corollary 4.4. For any $0 \leq i \leq \ell$ the following are equal to $h_{\ell-i}^{+}(\Phi, m)$ :

(i) the number of points in $D_{m}(\Phi)$ which lie in $i$ walls of $p \overline{A_{\circ}}$ and

(ii) the number of $w \in W_{m}(\Phi)$ such that $i$ walls of $w A_{\circ}$ of the form $H_{\alpha, m}$ do not separate $w A_{\circ}$ from $A_{\circ}$.

Proof: By Lemma 4.1, the number of orbits $x \in O_{m}(\Phi)$ with rank $r(x)=i$ is equal to the number of points in $D_{m}(\Phi)$ which lie in $i$ walls of $p \overline{A_{\circ}}$. The statement now follows by specializing Corollary 4.3 and recalling that $\Sigma_{m}^{m}=p \overline{A_{\circ}}$.

Remark 4.5. Part (ii) of Corollary 4.4 implies that $h_{\ell}^{+}(\Phi, m)$ is equal to the cardinality of $\check{Q} \cap(m h-1) A_{\circ}$. An argument similar to the one employed in [2, Remark 4.5] shows that $\check{Q} \cap(m h-1) A_{\circ}$ is equinumerous to $\check{Q} \cap(m h-h-1) \overline{A_{\circ}}$. Therefore from Theorem 1.1 (iii) we conclude that $h_{\ell}^{+}(\Phi, m)=N^{+}(\Phi, m-1)$. Since the reduced Euler characteristic 
$\tilde{\chi}\left(\Delta_{+}^{m}(\Phi)\right)$ of $\Delta_{+}^{m}(\Phi)$ is equal to $(-1)^{\ell-1} h_{\ell}\left(\Delta_{+}^{m}(\Phi)\right)$, it follows from the results of the next section that

$$
\tilde{\chi}\left(\Delta_{+}^{m}(\Phi)\right)=(-1)^{\ell-1} N^{+}(\Phi, m-1)
$$

if $\Phi$ has type $A, B$ or $C$ (see [7, (3.1)] for the corresponding property of $\Delta^{m}(\Phi)$ ).

The following conjecture is the positive analogue of [7, Conjecture 3.7].

Conjecture 4.6. For any crystallographic root system $\Phi$ and all $m \geq 1$ the complex $\Delta_{+}^{m}(\Phi)$ is pure $(\ell-1)$-dimensional and shellable and has reduced Euler characteristic equal to $(-1)^{\ell-1} N^{+}(\Phi, m-1)$.

In particular it is Cohen-Macaulay and has the homotopy type of a wedge of $N^{+}(\Phi, m-1)$ spheres of dimension $\ell-1$.

\section{The numbers $f_{i}(\Phi, m)$ and $f_{i}^{+}(\Phi, m)$}

Let $\Phi$ be a crystallographic root system of rank $\ell$ spanning the Euclidean space $V$. We define numbers $f_{i}(\Phi, m)$ and $f_{i}^{+}(\Phi, m)$ by the relations

$$
\sum_{i=0}^{\ell} f_{i-1}(\Phi, m)(x-1)^{\ell-i}=\sum_{i=0}^{\ell} h_{i}(\Phi, m) x^{\ell-i}
$$

and

$$
\sum_{i=0}^{\ell} f_{i-1}^{+}(\Phi, m)(x-1)^{\ell-i}=\sum_{i=0}^{\ell} h_{i}^{+}(\Phi, m) x^{\ell-i}
$$

respectively. Comparing to equation (5) we see that Conjecture 1.2 for the pair $(\Phi, m)$ is equivalent to the statement that

$$
f_{i-1}^{+}(\Phi, m)=f_{i-1}\left(\Delta_{+}^{m}(\Phi)\right)
$$

for all $i$, where $f_{i-1}\left(\Delta_{+}^{m}(\Phi)\right)$ is as in Section 2. We will give a combinatorial interpretation to the numbers $f_{i-1}(\Phi, m)$ and $f_{i-1}^{+}(\Phi, m)$ as follows. For $0 \leq k \leq \ell$ we denote by $\mathcal{F}_{k}(\Phi, m)$ the collection of $k$-dimensional (nonempty) sets of the form

$$
\bigcap_{(\alpha, r) \in \Phi^{+} \times\{0,1, \ldots, m\}} \tilde{H}_{\alpha, r}
$$

where $\tilde{H}_{\alpha, r}$ can be

$$
\begin{cases}H_{\alpha, 0}^{+}, & \text {if } r=0, \\ H_{\alpha, m}^{-}, H_{\alpha, m}^{+} \text {or } H_{\alpha, m}, & \text { if } r=m, \\ H_{\alpha, r}^{-} \text {or } H_{\alpha, r}^{+}, & \text {if } 1 \leq r<m\end{cases}
$$

and $H_{\alpha, r}^{-}$and $H_{\alpha, r}^{+}$denote the two open half-spaces in $V$ defined by the inequalities $(\alpha, x)<$ $r$ and $(\alpha, x)>r$, respectively. Observe that each element of $\mathcal{F}_{k}(\Phi, m)$ is dominant. We 包 Springer 
also denote by $\mathcal{F}_{k}^{+}(\Phi, m)$ the elements of $\mathcal{F}_{k}(\Phi, m)$ which are bounded subsets of $V$ or, equivalently, the sets of the form (11) with $\tilde{H}_{\sigma_{i}, m}=H_{\sigma_{i}, m}^{-}$or $H_{\sigma_{i}, m}$ for $1 \leq i \leq \ell$. In the special case $m=1$ part (iii) of the following theorem is the content of Remark 5.10 (v) in [6].

Theorem 5.1. For any irreducible crystallographic root system $\Phi$ and all $m \geq 1$ and $0 \leq$ $k \leq \ell$ the number $f_{k-1}(\Phi, m)$ counts

(i) pairs $(R, S)$ where $R$ is a dominant region of $\mathcal{A}_{\Phi}^{m}$ and $S$ is a set of $\ell-k$ walls of $R$ of the form $H_{\alpha, m}$ which separate $R$ from $A_{\circ}$,

(ii) pairs $(\mathcal{I}, T)$ where $\mathcal{I}$ is a geometric chain of filters in $\Phi^{+}$of length $m$ and $T$ is a set of $\ell-k$ indecomposable roots of rank $m$ with respect to $\mathcal{I}$ and

(iii) the elements of $\mathcal{F}_{k}(\Phi, m)$.

Proof: From (8) we have

$$
f_{k-1}(\Phi, m)=\sum_{i=0}^{k} h_{i}(\Phi, m)\left(\begin{array}{l}
\ell-i \\
\ell-k
\end{array}\right)
$$

which cleary implies (i) and (ii) (see Theorem 2.5). To complete the proof it suffices to give a bijection from the set $\mathcal{R}_{k}(\Phi, m)$ of pairs $(R, S)$ which appear in (i) to $\mathcal{F}_{k}(\Phi, m)$. Given such a pair $\tau=(R, S)$ let $g(\tau)$ be the intersection (11), where $\tilde{H}_{\alpha, r}$ is chosen so that $R \subseteq \tilde{H}_{\alpha, r}$ unless $r=m$ and $H_{\alpha, r} \in S$, in which case $\tilde{H}_{\alpha, r}=H_{\alpha, r}$. Let $S=\left\{H_{\alpha_{1}, m}, H_{\alpha_{2}, m}, \ldots, H_{\alpha_{\ell-k}, m}\right\}$ and let $F_{S}$ be the intersection of the hyperplanes in $S$. It follows from [2, Corollary 3.14] that $S$ is a proper subset of the set of walls of an alcove of $\widetilde{\mathcal{A}}_{\Phi}$ and hence that $F_{S}$ is nonempty and $k$-dimensional. To show that $g(\tau)$ is nonempty and $k$-dimensional, so that $g: \mathcal{R}_{k}(\Phi, m) \rightarrow$ $\mathcal{F}_{k}(\Phi, m)$ is well defined, we need to show that $F_{S}$ is not contained in any hyperplane $H_{\alpha, r}$ with $\alpha \in \Phi^{+}$and $0 \leq r \leq m$ other than those in $S$. So suppose that $F_{S} \subseteq H_{\alpha, r}$ with $\alpha \in \Phi^{+}$ and $r \geq 0$. Then there are real numbers $\lambda_{1}, \lambda_{2}, \ldots, \lambda_{\ell-k}$ such that

$$
\alpha=\lambda_{1} \alpha_{1}+\lambda_{2} \alpha_{2}+\cdots+\lambda_{\ell-k} \alpha_{\ell-k}
$$

and $r=m\left(\lambda_{1}+\lambda_{2}+\cdots+\lambda_{\ell-k}\right)$. Observe that the $\alpha_{i}$ are minimal elements of the last filter in the geometric chain of filters in $\Phi^{+}$corresponding to $R$ and hence that they form an antichain in $\Phi^{+}$, meaning a set of pairwise incomparable elements. It follows from the first main result of [16] (see the proof of [3, Corollary 6.2]) that the coefficients $\lambda_{i}$ in (12) are nonnegative integers. Hence either $r>m$ or $\alpha=\alpha_{i}$ and $r=m$ for some $i$, so that $H_{\alpha, r} \in S$.

To show that $g$ is a bijection we will show that given $F \in \mathcal{F}_{k}(\Phi, m)$ there exists a unique $\tau \in \mathcal{R}_{k}(\Phi, m)$ with $g(\tau)=F$. Let $\left(\varpi_{1}^{\vee}, \varpi_{2}^{\vee}, \ldots, \varpi_{\ell}^{\vee}\right)$ be the linear basis of $V$ which is dual to $\Pi$, in the sense that

$$
\left(\sigma_{i}, \varpi_{j}^{\vee}\right)=\delta_{i j}
$$

Observe that if $g(\tau)=F$ with $\tau=(R, S), x$ is a point in $F$ and $\epsilon_{i}$ are sufficiently small positive numbers then

$$
x+\sum_{i=1}^{\ell} \epsilon_{i} \varpi_{i}^{\vee} \in R .
$$


Since regions of $\mathcal{A}_{\Phi}^{m}$ are pairwise disjoint this implies uniqueness of $R$, and hence of $\tau$. To prove the existence let $R$ be the unique region of $\mathcal{A}_{\Phi}^{m}$ defined by (13). Equivalently $R$ can be obtained by replacing all hyperplanes of the form $H_{\alpha, m}$ in the intersection (11) defining $F$ by $H_{\alpha, m}^{+}$. It suffices to show that any such hyperplane $H_{\alpha, m}$ is a wall of $R$ since then, if $S$ is the set of hyperplanes of the form $H_{\alpha, m}$ which contain $F$ then $\tau=(R, S) \in \mathcal{R}_{k}(\Phi, m)$ and $g(\tau)=F$.

Suppose on the contrary that $H_{\alpha, m} \supseteq F$ is not a wall of $R$. It follows from Theorem 2.5 that $\alpha$ is not indecomposable of rank $m$ with respect to the geometric chain $\mathcal{I}$ of filters $\Phi^{+}=\mathcal{I}_{0} \supseteq \mathcal{I}_{1} \supseteq \cdots \supseteq \mathcal{I}_{m}$ corresponding to $R$ and hence that one can write $\alpha=\beta+\gamma$ for some $\beta \in \mathcal{I}_{i}, \gamma \in \mathcal{I}_{j}$ with $i+j=m$. Since $(\alpha, x)=m$ for $x \in F$ and $(\beta, x)>i$ and $(\gamma, x)>j$ hold for $x \in R$, so that $(\beta, x) \geq i$ and $(\gamma, x) \geq j$ hold for $x \in F$, we must have $(\beta, x)=i$ and $(\gamma, x)=j$ for $x \in F$. However one of $i, j$ must be less than $m$ and this contradicts the fact that $F$ can be contained in $H_{\alpha, r}$ for $\alpha \in \Phi^{+}$only if $r=m$.

The proof of the next theorem is entirely similar to that of Theorem 5.1 and is omitted.

Theorem 5.2. For any irreducible crystallographic root system $\Phi$ and all $m \geq 1$ and $0 \leq$ $k \leq \ell$ the number $f_{k-1}^{+}(\Phi, m)$ counts

(i) pairs $(R, S)$ where $R$ is a dominant bounded region of $\mathcal{A}_{\Phi}^{m}$ and $S$ is a set of $\ell-k$ walls of $R$ of the form $H_{\alpha, m}$ which do not separate $R$ from $A_{\circ}$,

(ii) pairs $(\mathcal{J}, T)$ where $\mathcal{J}$ is a positive geometric chain of ideals in $\Phi^{+}$of length $m$ and $T$ is a set of $\ell-k$ indecomposable roots of rank $m$ with respect to $\mathcal{J}$ and

(iii) the elements of $\mathcal{F}_{k}^{+}(\Phi, m)$.

The reader is invited to use part (iii) of Theorems 5.1 and 5.2 as well as Figure 1 to verify that $f_{-1}=1, f_{0}=8, f_{1}=12, f_{-1}^{+}=1, f_{0}^{+}=6$ and $f_{1}^{+}=7$ when $\Phi=A_{2}$ and $m=2$. It should be clear that apart from the necessary modifications in the statements of part (i), Theorems 5.1 and 5.2 are also valid when $\Phi$ is reducible.

Lemma 5.3. For any crystallographic root system $\Phi_{I}$ and all $m \geq 1$ and $0 \leq k \leq \ell$ we have

$$
f_{k-1}\left(\Phi_{I}, m\right)=\sum_{J \subseteq I} f_{k-|J|-1}^{+}\left(\Phi_{I \backslash J}, m\right)
$$

Proof: For $J \subseteq I$ let $V_{J}$ be the linear span of the simple roots indexed by the elements of $J$ and let $p_{J}: V_{I} \rightarrow V_{I \backslash J}$ be the orthogonal projection onto $V_{I \backslash J}$. We define the simple part of $F \in \mathcal{F}_{k}\left(\Phi_{I}, m\right)$ as the set of indices $j \in I$ such that $F \subseteq H_{\sigma_{j}, m}^{+}$. Observe that if $J$ is the simple part of $F$ then for $\alpha \in \Phi^{+}$and $x \in F$ we have

$$
\begin{array}{ll}
(\alpha, x)=\left(\alpha, p_{J}(x)\right), & \text { if } \alpha \in \Phi_{I \backslash J} \\
(\alpha, x)>m, & \text { otherwise. }
\end{array}
$$

It follows that $p_{J}$ induces a bijection from the set of elements of $\mathcal{F}_{k}\left(\Phi_{I}, m\right)$ with simple part $J$ to $\mathcal{F}_{k-|J|}^{+}\left(\Phi_{I \backslash J}, m\right)$. Hence counting the elements of $\mathcal{F}_{k}\left(\Phi_{I}, m\right)$ according to their simple part proves the lemma. 
The same type of argument as that in the next corollary appears in the proof of [12, Proposition 6.1].

Corollary 5.4. If for some pair $(\Phi, m)$ every parabolic subsystem of $\Phi$ satisfies (6) and we have $h_{i}(\Phi, m)=h_{i}\left(\Delta^{m}(\Phi)\right)$ for all $i$ then we have $h_{i}^{+}(\Phi, m)=h_{i}\left(\Delta_{+}^{m}(\Phi)\right)$ for all $i$ as well.

Proof: From the assumption we have $f_{k-1}(\Phi, m)=f_{k-1}\left(\Delta^{m}(\Phi)\right)$ for all $k$. Equation (6) and Lemma 5.3 imply that $f_{k-1}^{+}(\Phi, m)=f_{k-1}\left(\Delta_{+}^{m}(\Phi)\right)$ for all $k$ via Möbius inversion on the set of pairs $(k, I)$ partially ordered by letting

$$
(l, J) \leq(k, I) \text { if and only if } J \subseteq I \text { and } k-l=|I \backslash J| .
$$

This is equivalent to the conclusion of the corollary.

Corollary 5.5. Conjecture 1.2 holds for root systems of type $A, B$ or $C$ and any $m \geq 1$ and for all root systems when $m=1$.

Proof: This follows from Lemma 2.4, the previous corollary and the fact that the equality $h_{i}(\Phi, m)=h_{i}\left(\Delta^{m}(\Phi)\right)$ can be checked case by case from the explicit formulas given in $[2,7,18]$ in the cases under consideration.

We conclude this section with a combinatorial interpretation to $f_{k-1}^{+}(\Phi, m)$ similar to those provided in parts (i) and (ii) of Theorem 5.2.

Theorem 5.6. For any irreducible crystallographic root system $\Phi$ and all $m \geq 1$ and $0 \leq$ $k \leq \ell$ the number $f_{k-1}^{+}(\Phi, m)$ counts

(i) pairs $(R, S)$ where $R$ is a dominant region of $\mathcal{A}_{\Phi}^{m}$ and $S$ is a set of $\ell-k$ walls of $R$ of the form $H_{\alpha, m}$ which separate $R$ from $A_{\circ}$ such that $S$ contains all such walls of $R$ with $\alpha \in \Pi$ and

(ii) pairs $(\mathcal{I}, T)$ where $\mathcal{I}$ is a geometric chain of filters in $\Phi^{+}$of length $m$ and $T$ is a set of $\ell-k$ indecomposable roots of rank $m$ with respect to $\mathcal{I}$ which contains all simple indecomposable roots of rank $m$ with respect to $\mathcal{I}$.

Proof: The sets in (i) and (ii) are equinumerous by Theorem 2.5. To complete the proof one can argue that the map $g$ in the proof of Theorem 5.1 restricts to a bijection from the set in (i) to $\mathcal{F}_{k}^{+}(\Phi, m)$. Alternatively, arguing as in the proof of Corollary 5.4, it suffices to show that

$$
f_{k-1}\left(\Phi_{I}, m\right)=\sum_{J \subseteq I} g_{k-|J|-1}^{+}\left(\Phi_{I \backslash J}, m\right)
$$

where $\Phi=\Phi_{I}$ and $g_{k-1}^{+}(\Phi, m)$ denotes the cardinality of the set of pairs, say $\mathcal{G}_{k}^{+}(\Phi, m)$, which appears in (ii). Let $\mathcal{G}_{k}\left(\Phi_{I}, m\right)$ denote the set of pairs defined in (ii) of the statement of Theorem 5.1. For $(\mathcal{I}, T) \in \mathcal{G}_{k}\left(\Phi_{I}, m\right)$ call the set of simple roots which are indecomposable of rank $m$ with respect to $\mathcal{I}$ and are not contained in $T$ the simple part of $(\mathcal{I}, T)$ and for 
any $J \subseteq I$ denote by $\Lambda_{J}$ the order filter of roots $\alpha \in \Phi_{I}^{+}$for which $\sigma \leq \alpha$ for some $\sigma \in J$. It is straightforward to check from the definitions that the map which sends a pair $(\mathcal{I}, T)$ to $\left(\mathcal{I} \backslash \Lambda_{J}, T\right)$, where $\mathcal{I} \backslash \Lambda_{J}$ denotes the chain obtained from $\mathcal{I}$ by removing $\Lambda_{J}$ from each filter of $\mathcal{I}$, induces a bijection from the set of elements of $\mathcal{G}_{k}\left(\Phi_{I}, m\right)$ with simple part $J$ to $\mathcal{G}_{k-|J|}^{+}\left(\Phi_{I \backslash J}, m\right)$. Therefore counting the elements of $\mathcal{G}_{k}\left(\Phi_{I}, m\right)$ according to their simple part gives the desired equality.

\section{Classical types and the case $m=1$}

In this section we compute the numbers $h_{i}^{+}(\Phi, m)$ and $f_{i-1}^{+}(\Phi, m)$ in the cases of the classical root systems.

Proposition 6.1. The number $h_{i}^{+}(\Phi, m)$ is equal to

$$
\begin{array}{ll}
\frac{1}{i+1}\left(\begin{array}{c}
n-1 \\
i
\end{array}\right)\left(\begin{array}{c}
m n-2 \\
i
\end{array}\right), & \text { if } \Phi=A_{n-1}, \\
\left(\begin{array}{c}
n \\
i
\end{array}\right)\left(\begin{array}{c}
m n-1 \\
i
\end{array}\right), & \text { if } \Phi=B_{n} \text { or } C_{n}, \\
\left(\begin{array}{l}
n \\
i
\end{array}\right)\left(\begin{array}{c}
m(n-1)-1 \\
i
\end{array}\right)+\left(\begin{array}{c}
n-2 \\
i-2
\end{array}\right)\left(\begin{array}{c}
m(n-1) \\
i
\end{array}\right), & \text { if } \Phi=D_{n} .
\end{array}
$$

Proof: The proof can be obtained from that of [2, Proposition 5.1] by replacing the quantity $m h+1$ by $m h-1$ and using Theorem 1.3 (iii) instead of [2, Theorem 1.2 (ii)].

The following corollary is a straightforward consequence of Proposition 6.1 and equation (9).

Corollary 6.2. The number $f_{k-1}^{+}(\Phi, m)$ is equal to

$$
\begin{array}{ll}
\frac{1}{k+1}\left(\begin{array}{c}
n-1 \\
k
\end{array}\right)\left(\begin{array}{c}
m n+k-1 \\
k
\end{array}\right), & \text { if } \Phi=A_{n-1}, \\
\left(\begin{array}{l}
n \\
k
\end{array}\right)\left(\begin{array}{c}
m n+k-1 \\
k
\end{array}\right), & \text { if } \Phi=B_{n} \text { or } C_{n}, \\
\left(\begin{array}{l}
n \\
k
\end{array}\right)\left(\begin{array}{c}
m(n-1)+k-1 \\
k
\end{array}\right)+\left(\begin{array}{l}
n-2 \\
k-2
\end{array}\right)\left(\begin{array}{c}
m(n-1)+k-2 \\
k
\end{array}\right), & \text { if } \Phi=D_{n} .
\end{array}
$$

In the case $m=1$, the following corollary for $\Phi=A_{n-1}$ and $\Phi=B_{n}, C_{n}$ is a special case of $[5,(34)]$ and $[5,(46)]$, respectively. 
Corollary 6.3. The number $f_{k-1}\left(\Delta_{+}^{m}(\Phi)\right)$ is equal to

$$
\begin{aligned}
\frac{1}{k+1}\left(\begin{array}{c}
n-1 \\
k
\end{array}\right)\left(\begin{array}{c}
m n+k-1 \\
k
\end{array}\right), & \text { if } \Phi=A_{n-1}, \\
\left(\begin{array}{l}
n \\
k
\end{array}\right)\left(\begin{array}{c}
m n+k-1 \\
k
\end{array}\right), & \text { if } \Phi=B_{n} \text { or } C_{n} .
\end{aligned}
$$

Moreover

$$
f_{k-1}\left(\Delta_{+}\left(D_{n}\right)\right)=\left(\begin{array}{l}
n \\
k
\end{array}\right)\left(\begin{array}{c}
n+k-2 \\
k
\end{array}\right)+\left(\begin{array}{l}
n-2 \\
k-2
\end{array}\right)\left(\begin{array}{c}
n+k-3 \\
k
\end{array}\right) .
$$

Proof: Combine Corollaries 5.5 and 6.2.

Remark 6.4. The number of positive filters in $\Phi^{+}$with $i$ minimal elements has been computed for the exceptional root systems by Victor Reiner as shown in Table 1.

Proof of Theorem 1.5: We will prove the statement of the theorem without the assumption that $\Phi$ is irreducible. Let $\ell$ be the rank of $\Phi=\Phi_{I}$. We write $h_{k}\left(\Phi_{I}\right)$ instead of $h_{k}\left(\Phi_{I}, 1\right)$, so that $h_{\ell-k}\left(\Phi_{I}\right)$ counts the filters in $\Phi_{I}^{+}$with $k$ minimal elements as well as the ideals in $\Phi_{I}^{+}$with $k$ maximal elements. Let $\widetilde{h}_{k}^{+}(\Phi)$ denote the number of positive filters in $\Phi^{+}$with $k$ minimal elements. Counting filters in $\Phi_{I}^{+}$by the set of simple roots they contain gives

$$
h_{\ell-k}\left(\Phi_{I}\right)=\sum_{J \subseteq I} \widetilde{h}_{k-|J|}^{+}\left(\Phi_{I \backslash J}\right)
$$

Similarly, counting ideals in $\Phi_{I}^{+}$by the set of simple roots they do not contain gives

$$
h_{\ell-k}\left(\Phi_{I}\right)=\sum_{J \subseteq I} h_{\ell-|J|-k}^{+}\left(\Phi_{I \backslash J}\right) .
$$

Since it is known [13] that $h_{\ell-k}\left(\Phi_{I}\right)=h_{k}\left(\Phi_{I}\right)$ the previous relation can also be written as

$$
h_{\ell-k}\left(\Phi_{I}\right)=\sum_{J \subseteq I} h_{k-|J|}^{+}\left(\Phi_{I \backslash J}\right)
$$

Table 1 The numbers $h_{i}^{+}(\Phi)$ for the exceptional root systems

\begin{tabular}{cllllllll}
\hline \multicolumn{1}{c}{$i$} & 0 & 1 & 2 & 3 & 4 & 5 & 6 & 7 \\
\hline$\Phi=G_{2}$ & 1 & 4 & & & & & & \\
$\Phi=F_{4}$ & 1 & 20 & 35 & 10 & & & & \\
$\Phi=E_{6}$ & 1 & 30 & 135 & 175 & 70 & 7 & & \\
$\Phi=E_{7}$ & 1 & 56 & 420 & 952 & 770 & 216 & 16 & \\
$\Phi=E_{8}$ & 1 & 112 & 1323 & 4774 & 6622 & 3696 & 770 & 44 \\
\hline
\end{tabular}


Comparing (14) and (15) and using Möbius inversion as in Section 5 gives $\widetilde{h}_{k}^{+}\left(\Phi_{J}\right)=h_{k}^{+}\left(\Phi_{J}\right)$ for all $J \subseteq I$, which is the statement of the theorem.

\section{Remarks}

1. The following reciprocity relation

$$
N^{+}(\Phi, m-1)=(-1)^{\ell} N(\Phi,-m)
$$

was observed by Fomin and Reading [7, (2.12)]. We will show that, as suggested by S. Fomin (private communication), this relation is in fact an instance of Ehrhart reciprocity. Let $i(n)$ be the cardinality of $\check{Q} \cap n \overline{A_{\circ}}$ for $n \in \mathbb{N}$. It is clear that the vertices of the simplex $\overline{A_{\circ}}$ have rational coordinates in the basis $\Pi$ of $V$ of simple roots, hence also in the basis $\Pi^{\vee}=\{2 \alpha /(\alpha, \alpha): \alpha \in \Pi\}$ of $V$. Therefore the function $i(n)$ is the Ehrhart quasipolynomial of $\overline{A_{\circ}}$ with respect to the lattice $\check{Q}$ (see [17, Section 4.6] for an introduction to the theory of Ehrhart quasi-polynomials). Ehrhart reciprocity [17, Theorem 4.6.26] implies that

$$
(-1)^{\ell} i(-n)=\#\left(\check{Q} \cap n A_{\circ}\right)
$$

for $n \in \mathbb{N}$ and hence, setting $n=m h-1$ and consulting [2, Theorem 1.1], that

$$
(-1)^{\ell} N(\Phi,-m)=\#\left(\check{Q} \cap(m h-1) A_{\circ}\right) .
$$

Remark 4.5 asserts that

$$
\#\left(\check{Q} \cap(m h-1) A_{\circ}\right)=N^{+}(\Phi, m-1)
$$

and hence (16) holds.

2. It would be interesting to give combinatorial proofs of the formulas in Corollary 6.3 directly from the description of the relevant complexes given in [7, 9, 18] and Section 2.

3. After this paper was completed the following came to our attention. (i) The numbers $\widetilde{h}_{i}^{+}(\Phi)$ of positive filters in $\Phi^{+}$with $i$ minimal elements are also discussed and partially computed in [14, Section 3]. (ii) Theorem 2.7 in [8] implies that Lemma 2.4 is valid for all pairs $(\Phi, m)$. In view of (ii) and the equality $h_{i}(\Phi, m)=h_{i}\left(\Delta^{m}(\Phi)\right)($ see $[2,7,8])$ when $\Phi=D_{n}$, it follows from Corollary 5.4 that Conjecture 1.2 is also valid for root systems of type $D$ and arbitrary $m$.

Acknowledgments We are grateful to Sergey Fomin and Nathan Reading for making their work [7] available to us. We also thank Sergey Fomin for useful discussions and Victor Reiner for providing the data of Table 1 as well as the software to confirm it.

\section{References}

1. C.A. Athanasiadis, Generalized Catalan numbers, Weyl groups and arrangements of hyperplanes, Bull. London Math. Soc. 36 (2004), 294-302.

2. C.A. Athanasiadis, On a refinement of the generalized Catalan numbers for Weyl groups, Trans. Amer. Math. Soc. 357 (2005), 179-196. 
3. C.A. Athanasiadis and V. Reiner, Noncrossing partitions for the group $\mathrm{D}_{\mathrm{n}}$, SIAM J. Discrete Math. 18 (2004), 397-417.

4. N. Bourbaki, Lie Groups and Lie Algebras, Chapters 4-6, Springer-Verlag, Berlin, 2002.

5. F. Chapoton, Enumerative properties of generalized associahedra, Sémin. Loth. de Combinatoire $\mathbf{5 1}$ (2004), Article B51b, 16pp (electronic).

6. S. Fomin and N. Reading, Root systems and generalized associahedra, in Geometric Combinatorics, IAS/Park City Mathematics Series (to appear).

7. S. Fomin and N. Reading, Generalized cluster complexes and Coxeter combinatorics, incomplete preliminary draft of January 6, 2005, 30pp.

8. S. Fomin and N. Reading, Generalized cluster complexes and Coxeter combinatorics, Int. Math. Res. Not. 44 (2005), 2709-2757.

9. S. Fomin and A.V. Zelevinsky, $Y$-systems and generalized associahedra, Ann. of Math. 158 (2003), 977-1018.

10. M.D. Haiman, Conjectures on the quotient ring by diagonal invariants, J. Algebraic Combin. 3 (1994), $17-76$.

11. J.E. Humphreys, Reflection groups and Coxeter groups, Cambridge Studies in Advanced Mathematics 29, Cambridge University Press, Cambridge, England, 1990.

12. R. Marsh, M. Reineke and A. Zelevinsky, Generalized associahedra via quiver representations, Trans. Amer. Math. Soc. 355 (2003), 4171-4186.

13. D.I. Panyushev, Ad-nilpotent ideals of a Borel subalgebra: generators and duality, J. Algebra 274 (2004), 822-846.

14. D.I. Panyushev, Two covering polynomials of a finite poset, with applications to root systems and adnilpotent ideals, preprint, 2005, 20pp (ArXiV preprint math.CO/0502386).

15. J.-Y. Shi, Alcoves corresponding to an affine Weyl group, J. London Math. Soc. 35 (1987), 42-55.

16. E. Sommers, $B$-stable ideals in the nilradical of a Borel subalgebra, Canad. Math. Bull. 48 (2005), 460-472.

17. R.P. Stanley, Enumerative Combinatorics, vol. 1, Wadsworth \& Brooks/Cole, Pacific Grove, CA, 1986; second printing, Cambridge University Press, Cambridge, 1997.

18. E. Tzanaki, Polygon dissections and some generalizations of cluster complexes, J. Combin. Theory Series $A$ (to appear). 